Swarthmore College

Works

$9-1-1996$

\title{
Measurement of pressure and assessment of cavitation for a 22.5-kHz intra-arterial angioplasty device
}

\author{
I. R. S. Makin \\ E. Carr Everbach \\ Swarthmore College, ceverba1@swarthmore.edu
}

Follow this and additional works at: https://works.swarthmore.edu/fac-engineering

Part of the Engineering Commons

Let us know how access to these works benefits you

\section{Recommended Citation}

I. R. S. Makin and E. Carr Everbach. (1996). "Measurement of pressure and assessment of cavitation for a 22.5-kHz intra-arterial angioplasty device". Journal Of The Acoustical Society Of America. Volume 100, Issue 3. 1855-1864. DOI: 10.1121/1.416005

https://works.swarthmore.edu/fac-engineering/63

This work is brought to you for free by Swarthmore College Libraries' Works. It has been accepted for inclusion in Engineering Faculty Works by an authorized administrator of Works. For more information, please contact myworks@swarthmore.edu. 


\title{
Measurement of pressure and assessment of cavitation for a $22.5-\mathrm{kHz}$ intra-arterial angioplasty device
}

\author{
Inder Raj S. Makin and E. Carr Everbach \\ Swarthmore College, Department of Engineering, Swarthmore, Pennsylvania 19081-1397
}

(Received 29 August 1995; accepted for publication 24 March 1996)

\begin{abstract}
This study was performed to understand better the mechanisms of action of an $(22.5 \mathrm{kHz})$ ultrasonic wire catheter device used to remove atheromatous plaque in diseased blood vessels (ultrasonic angioplasty). During a clinical procedure, the wire acts as an acoustic waveguide to transfer acoustic energy from a generator outside the body to the ball tip of the wire, which is inserted in the blood vessel. The acoustic field radiated by the vibrating ball tip (1.5- to 3.0-mm diameter), was mapped in a relatively large $(600 \mathrm{~L})$ water tank and compared to the field from a well-characterized simple source. A dipolelike radiation pattern due to the translating ball tip was observed. At low power settings, standing wave effects in a smaller cylindrical volume (200-mm diameter, 350-mm height), which was used to simulate anthropometric dimensions, increase relative to the larger tank measurements. The standing wave ratio is dependent upon the $\rho c$ characteristics of the medium and the dimensions of the volume, rather than on the absorption at this frequency. At high power settings of the device, cavitation at the tip of the wire was measured using a $20-\mathrm{MHz}$ passive cavitation detection scheme. (C) 1996 Acoustical Society of America.
\end{abstract}

PACS numbers: 43.80.Sh, 43.80.Gx, 43.35.Ei [FD]

\section{INTRODUCTION}

Intensive research efforts are underway to control the high incidence of coronary arterial disease and the occlusion of the peripheral vasculature among the aging human population in western societies. One approach in treatment is the development of instrumentation that invasively removes the obstruction in the diseased vessel. Removal of atheromatous plaque (angioplasty) is currently achieved by a wide array of physical means such as vessel dilating balloons, laser beams, mechanical tissue removal devices, as well as low-frequency ultrasonic wires. ${ }^{1,2}$ However, as mentioned in Ref. 1, the various methods of angioplasty exploit two basic physical techniques of tissue repair, namely "remodeling" of the lumen by compressing the cholesterol plaque and "removal" of the occluding material. Ultrasound angioplasty falls principally in the latter device category, whereby the blocking material is physically removed from the underlying normal lining of the blood vessel.

Compared to competing angioplasty techniques such as laser angioplasty and mechanical atherectomy devices, the ultrasonic technique affords advantages in terms of more controlled and selective removal of the diseased fibrotic and/or calcified tissue layer, while sparing the underlying normal layers. ${ }^{2-7}$ In addition, the particle size of the ablated debris is sufficiently small to minimize the blockage of vessels downstream and is comparable to that produced by other therapeutic techniques. ${ }^{2,7}$ Hence the ultrasound modality entails comparable risks due to embolization (blockage) in the circulation comparable to that of current angioplasty techniques.

The description and the speculated mechanisms of the ultrasonic angioplasty techniques have so far been described mainly in the medical literature. The speculated mechanisms of action of these devices are based on the experience gained in a clinical setting for in vitro experiments, or in vivo animal or human studies. Only recently has a theoretical analysis of low-frequency ultrasonic devices such as the ones used for angioplasty been presented by Nyborg. ${ }^{8}$ It is believed that bubble growth and collapse caused by the acoustic field around the oscillating ball tip of the wire is a mechanism of action of the device. ${ }^{2,8,9}$ However, cavitation in the ultrasonic angioplasty system could possibly result from a combination of effects, namely, the acoustic field due to vibration of the wire tip as well as the pressure gradients around the tip caused by the hydrodynamic flow field. The hydrodynamics of a sphere moving through water will briefly be considered in Sec. III and the Appendix. The aim of this research is to perform direct pressure measurements of the acoustic fields emitted by the ultrasonic source in order to attain a better understanding of the radiation pattern of the source and, in addition, to gain a better understanding of the mechanisms of action of the device.

\section{MATERIALS AND METHODS}

\section{A. Description of the device}

The device under investigation [Alliger Ultrasonic Wire System (AUWS), MISONIX Inc., Farmingdale, NY] is being developed for a technique of disruption of atherosclerotic plaque and thrombi in the coronary and peripheral blood vessels by using sound energy. The functional components of the system are: a generator, convertor, dampner, and the wire, as shown in Fig. 1. The generator supplies a highvoltage signal at $22.5 \mathrm{kHz}$ to the piezoelectric crystals (PZT4) placed in the convertor. The acoustic energy radiated from the piezo elements is guided through a tapered stainless steel horn and conducted further into a titanium wire of variable length and thickness. The wire terminates in a ball tip and is encased in a Teflon ${ }^{\circledR}$ sheath. In order to restrict the transverse whipping of the wire during operation, a fluid 


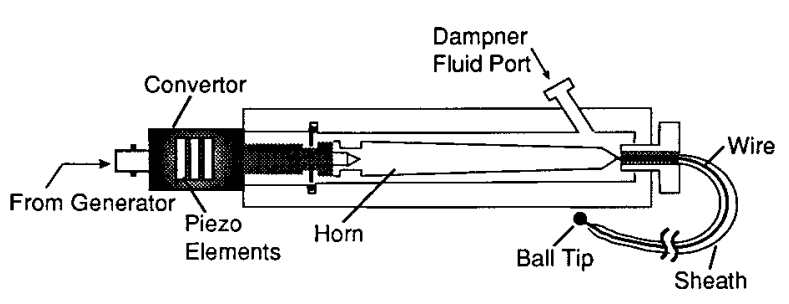

FIG. 1. Schematic of AUWS.

(preferably of high viscosity) is pumped under pressure (50$100 \mathrm{psi}$ ) through the dampner port and under the sheath covering the wire (see Fig. 1). The diameters of the ball tips for the wires used in our experiments were 1.98 and $2.46 \mathrm{~mm}$; the wires' respective lengths were 445 and $660 \mathrm{~mm}$. The measured amplitudes of displacement in the longitudinal direction for the two wires at full operational setting of the device were given by the manufacturer to be about 100 and $65 \mu \mathrm{m}$, respectively. The two wires described above and investigated here will for simplicity be referred to as wires 1 and 2 , respectively. The ball tip of wire 1 is mushroom shaped.

\section{B. Characteristics of the wire source}

During operation of the device, sound is radiated into the surrounding fluid mainly by the axial translatory motion of the ball tip of the wire. Since the axial movement of the ball tip $(\approx 100 \mu \mathrm{m})$, as well as the radius of the ball tip $(\approx 1.3$ $\mathrm{mm})$, are both much less than the wavelength of the driving frequency in water $(\lambda=66.6 \mathrm{~mm}$ for $f=22.5 \mathrm{kHz}, c=1500$ $\mathrm{m} / \mathrm{s}$ ), the field radiated by the translatory movement of the ball tip (see Fig. 2) may be approximated by that of a dipole. $^{8}$ Assuming that the radiation of sound from the ball tip is dipolelike, a theoretical estimation of negative acoustic pressures occurring at the tip can be made in order to predict whether or not these pressures are sufficient to cause acoustic cavitation. For a ball tip of nominal radius $a=1.3 \mathrm{~mm}$ having a maximum displacement $d=100 \mu \mathrm{m}$, the acoustic pressure amplitudes expected at the wire tip are about $600 \mathrm{kPa}-$ sufficient to cause cavitation at the operating frequency of $22.5 \mathrm{kHz} .{ }^{8,10,11}$

As mentioned earlier, violent collapse of bubbles around the tip of the wire is a possible mechanism of action of this device. Cavitation could result during operation of the device at high settings caused by acoustic pressure fields, as well as the hydrodynamic activity around the wire tip. A brief discussion of the possible importance of hydrodynamic phe-

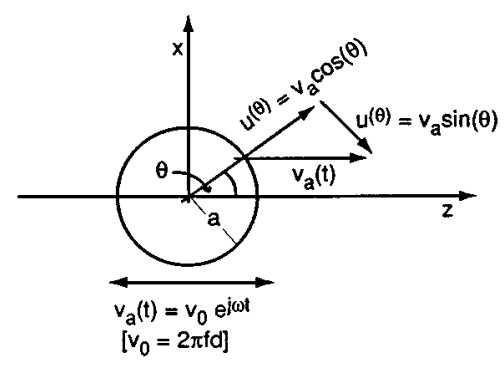

FIG. 2. Schematic of a translating sphere.

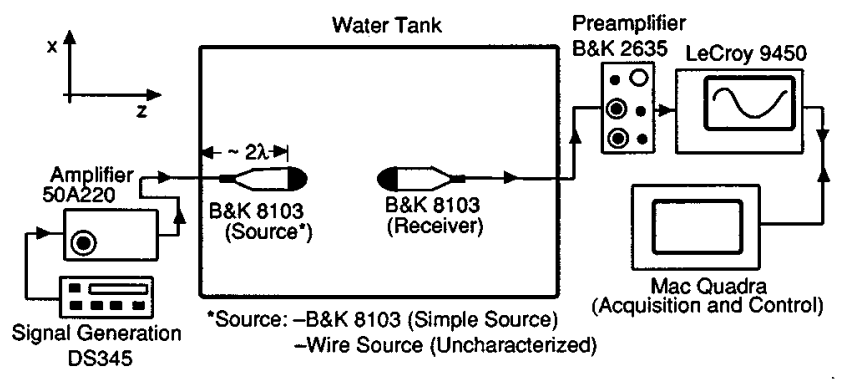

FIG. 3. Measurement setup for excitation of source and acquisition of received signal.

nomena in causing cavitation in the vicinity of the ball tip is given in the Appendix.

The comparison of our experiments with theoretical predictions from a model of a translating sphere operating as a dipole source can be qualitative at best since the model assumes a free spherical body translating only in the $z$ direction (refer to Fig. 2). In reality, however, a spherelike ball tip is attached to a wire which has a tendency to whip transversely.

\section{Field measurements in a large volume}

Measurement of the acoustic field from the ultrasonic wire when it radiates in a relatively free field was performed in the largest available tank, which measured $914 \times 812 \times 812$ $\mathrm{mm}$ and was fabricated from $12.5-\mathrm{mm}$ acrylic. However, significant standing waves were still observed in the tank because of the relatively long wavelength of the signal and the almost negligible attenuation of sound in water at $22.5 \mathrm{kHz}$. The approach for assessing the field due to the unknown source, i.e., the ultrasonic wire, was to compare its pattern with the field due to a known simple source [Brüel \& Kjær $(\mathrm{B} \& \mathrm{~K})$ model 8103] operated at the same frequency, and located at the same position as the wire in the tank. The tank in each case was filled with water to the same level.

The experiments were controlled from a Macintosh Quadra 700 computer with a National Instruments GPIB interface. In addition, the AUWS generator was activated from the computer by a NBMIO-16X D/A card. The programming environment used to control various instruments and acquisition of data was LabVIEW (National Instruments). A schematic of the experimental setup is shown in Fig. 3. Measurement of the acoustic fields was performed by raster scanning the horizontal and vertical planes symmetrically about the source under investigation. The receiver used was a second B\&K 8103 hydrophone, which, according to the manufacturer, is a broadband, omnidirectional receiver in the $100-\mathrm{Hz}$ to $120-\mathrm{kHz}$ range $(<1-\mathrm{dB}$ pressure deviation in the $x z$ and $y z$ planes). The receiver is mounted on a computercontrolled three-axis positioner (VELMEX Unislide lead screw with NF-90 controller) as close to the source as possible and aligned end-on to the source under investigation. The planes scanned were symmetric about the $z$ axis (see Fig. 3). Data points acquired for both the vertical and horizontal planes scanned the plane $150 \times 150 \mathrm{~mm}$ about the $z$ axis, at a resolution of $3 \mathrm{~mm}$. The resolution of $3 \mathrm{~mm}$ was observed to be adequate for sources radiating a signal of 66.6-mm wavelength. The signal received at the B\&K 8103 
hydrophone was amplified and filtered by a charge preamplifier (B\&K model 2635) and recorded by a digital oscilloscope (LeCroy model 9450). The amplitude of the signal acquired by the scope was downloaded to the computer into a file.

As a first step, the accuracy of the B\&K 8103 transducer as a simple source radiating at $22.5 \mathrm{kHz}$ was tested. The source is pulsed with an eight-cycle toneburst at a pulse repetition frequency (PRF) of $5 \mathrm{~Hz}$. The received signal measured is the amplitude of the direct wave signal launched from the source, such that any reflections from the tank boundaries are gated out. The measured pressure decay with range for the B\&K 8103 source was in good comparison with the theoretically predicted pressure decay for a simple source Fig. 4(a). The scans in Fig. 4(b) and (c) show the smooth decay of pressure for the B\&K 8103 source in the horizontal and vertical planes, symmetric about the $z$ axis. The source is pulsed as described earlier.

The simple source ( $\mathrm{B} \& \mathrm{~K}$ 8103) is next driven in the continuous wave $(\mathrm{CW})$ mode and its pressure field is measured, again, in the horizontal and vertical planes symmetric about the $z$ axis. As in the case of the scans in the pulsed mode, the scanned planes extend from 21 to $171 \mathrm{~mm}$ in the $z$ direction, and from -75 to $75 \mathrm{~mm}$ in the $x$ or $y$ direction. The step size between each measurement point is $3 \mathrm{~mm}$.

The simple source is next replaced by the wire source. Although we expect a dipolelike radiation from this source, its field is complicated because of the transverse whipping of the wire. For these measurements, the wire is driven at very low power settings to minimize the influence on the field of the transverse motion of the wire. In addition, the low gain settings of the device allow the piezocrystals and the horn assembly to operate in their linear regimes. The acoustic fields for the two wires under investigation were measured in the form of horizontal and vertical planes extending between 12 and $171 \mathrm{~mm}$ along the $z$ axis and -75 to $75 \mathrm{~mm}$ along the $y$ and $x$ axes. Due to the design geometry of the sources, the receiver hydrophone can be brought closer to the source in the case of the wires as compared to the B\&K 8103 source used in the previous case.

Finally, for both the wires under investigation, the gain on the generator is turned up to a setting comparable to that used during a therapeutic procedure (dial settings of 13-14 on the generator). Measurement of acoustic pressures were made along the axis of the wire ( $z$ axis) from a range of 13 to $245 \mathrm{~mm}$ in step sizes of $2 \mathrm{~mm}$. The received signal in this case is the amplified broadband response of the B\&K 8103 transducer. The observed signal in the time domain is now erratic, the signal now having frequency components much higher than the $22.5-\mathrm{kHz}$ driving frequency. The recorded pressure amplitudes correspond to the peak-to-peak pressure at a particular point noted at each instant in time.

\section{Field measurements in a cylindrical tube}

In the clinical setting, the ultrasonic wire is introduced through a peripheral blood vessel and operated within the body. It is relevant, therefore, to investigate the manner in which the acoustic field of the device varies when it is operated in a small volume. The scenario of the wire catheteriz- ing a blood vessel in an extremity is approximated by a cylindrical "tube-in-a-tube" model as shown in Fig. 5. The model consists of an upright acrylic cylinder (inside diameter $203 \mathrm{~mm}, 355-\mathrm{mm}$ high, wall thickness $3.175 \mathrm{~mm}$ ), with a flexible polyurethane tube (inside diameter $12.5 \mathrm{~mm}$, $1.5-\mathrm{mm}$ wall thickness) affixed at its center along the cylinder's axis. Both ends of the cylinder are covered with 9.525mm-thick acrylic flanges. The inside tube and the cylinder can be filled with similar or different liquids.

The source is placed approximately $65 \mathrm{~mm}$ from the lower wall of the cylinder. The inner tube has an inside diameter of $12.5 \mathrm{~mm}$ in order to accommodate the receiver B\&K 8103 hydrophone. The measurement protocol followed for these series of experiments in the cylindrical tube is such that the acoustic fields for the simple B\&K 8103 source as well as the unknown wire source are scanned along the long axis of the source for up to $251 \mathrm{~mm}$ from the source. Measurements are first made with both the polyurethane tube and the cylinder filled with water up to $345 \mathrm{~mm}$. The measurements are then repeated with the cylinder filled with a mixture of 2 parts $99.9 \%$ Glycerin and 1 part water, for the simple source as well as the wires. For comparison of the fields from the B\&K simple source with the two wire sources investigated, the the gain setting of the generator for the wire device is kept low (dial settings $\approx 4$ ), in order to minimize the transverse whipping of the wires. The pressure amplitude as a function of axial range is determined from the received signal, which is low-pass filtered around $22.5 \mathrm{kHz}$.

The axial pressure fields of both the wires being investigated are also measured for high gain settings of the generator (dial setting 14-16). The liquid media in this case were: deionized, degassed water in the cylinder as well as the inner tube, or both the cylinder and the inner tube filled with the Glycerin solution. The cylinder is filled with Glycerin, the inner tube filled with Hexabrix ${ }^{\circledR}$, which is the dampning fluid for the device during clinical use.

\section{E. Evidence of acoustic cavitation}

During operation of the ultrasonic wire at high gain settings, significant jet formation can be observed originating from the ball tip of the wire. It is therefore relevant to detect the occurrence of cavitational activity, and then to investigate a correlation between the gain setting of the device and the resulting cavitational activity.

Cavitation is detected by using active and passive acoustic techniques, whereby a high-frequency $(20-30 \mathrm{MHz})$ focused transducer is used to detect the rapid growth and collapse of individual bubbles or bubble clouds in the region of the spatial pressure maximum in a focused acoustic field. ${ }^{12-14}$ The techniques described in Refs. $12-14$ are principally used to assess pressure thresholds for cavitation and detect cavitation events in a focused sound field from a piston source operating in the megahertz frequency range, conditions which are more common in diagnostic medical ultrasound. A 20-MHz focused transducer (Krautkramer Branson, narrow bandwidth series), having a nominal diameter of 6.3 $\mathrm{mm}$ and a focal length of $51 \mathrm{~mm}$, is used here to probe the region of the wire just distal to its ball tip first in the pulseecho mode (active cavitation detection). ${ }^{12}$ Subsequently the 
(a)
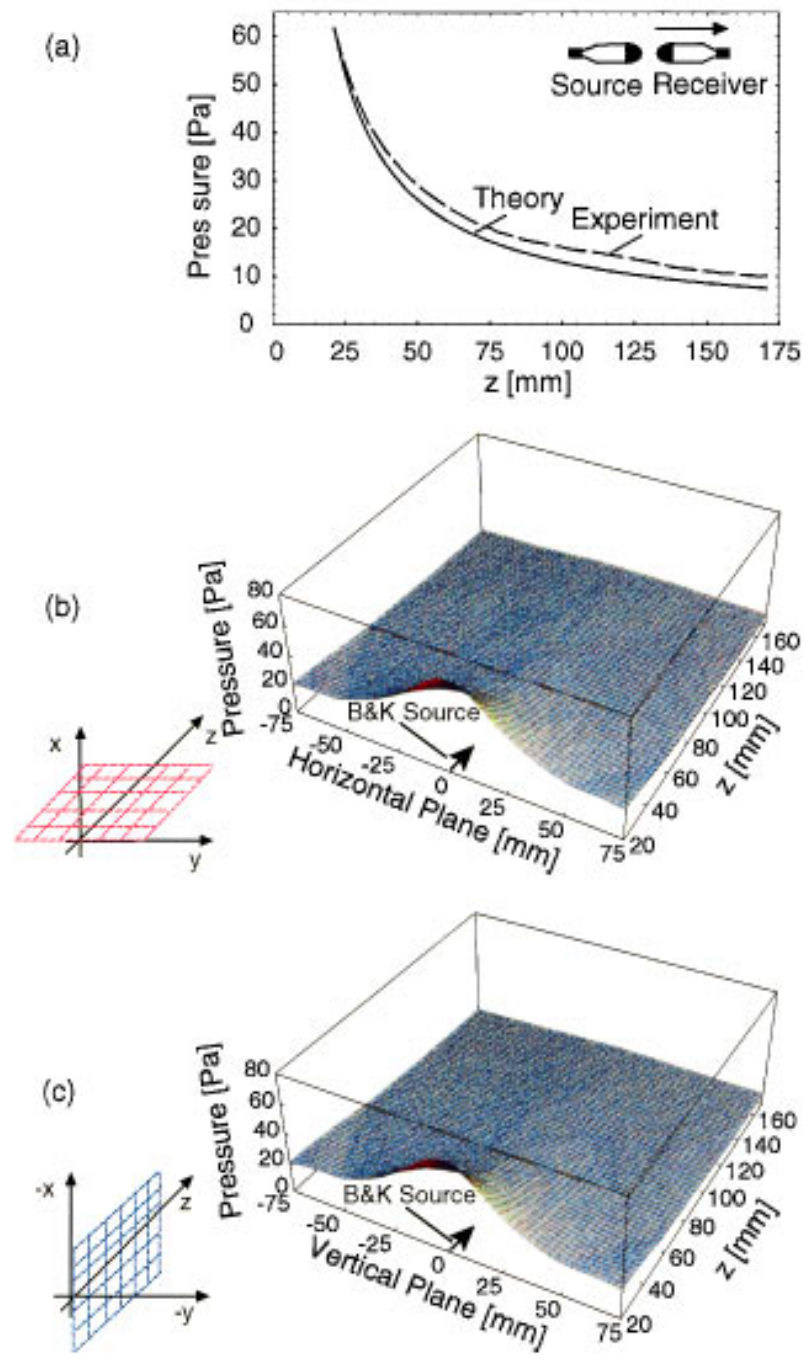

FIG. 4. Comparison of theory for a simple source with experiment [(a)], and field maps for vertical and horizontal planes [(b) and (c)], for the B\&K 8103 excited in the pulsed mode.

transducer recorded passively the $20 \mathrm{MHz}$ component of the broadband acoustic emissions of collapsing bubbles in the field due the ultrasonic wire. ${ }^{14}$

A schematic diagram of the setup for the detection of cavitation is shown in Fig. 6. Briefly, the ultrasonic wire is positioned in a cell measuring $100 \times 80 \times 70 \mathrm{~mm}$ such that the distal tip of the ball on the wire is at the focus of the $20-\mathrm{MHz}$ probe. The axis of the probe transducer is perpendicular to the axis of the wire. The cell is filled with deionized and degassed water. Since the cell is open to the atmosphere, the gas content of water attains equilibrium with the atmosphere prior to the experiment. The focus of the probe transducer is then moved beyond the ball tip by $1 \mathrm{~mm}$ along the $z$ axis (see Fig. 6), with the help of a three-axis micrometer positioning stage on which the probe transducer is mounted. Since even at maximum operational settings, the longitudinal translatory amplitudes of the ball tip cannot be greater than $100 \mu \mathrm{m}$, shifting of the wire tip by $1 \mathrm{~mm}$ from the focus ensures that the signal received by the probe transducer is not a result of reflections from the surface of the wire tip. Any signal output at the $20-\mathrm{MHz}$ transducer, therefore, is due to the violent

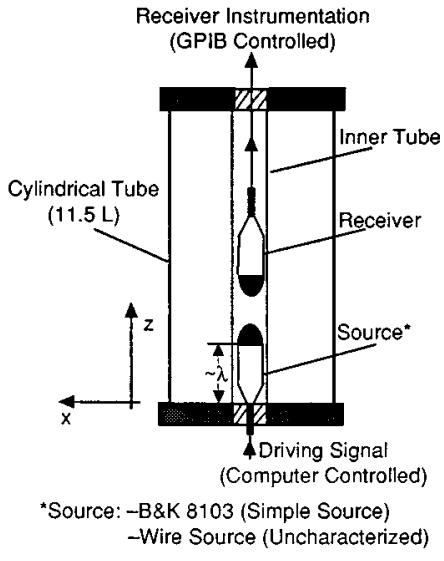

FIG. 5. Tube-in-tube model for field measurement in a bodylike space.

collapse of individual bubbles and/or bubble clouds just distal to the ball tip of the wire. A broad bandwidth needle hydrophone is used simultaneously to monitor the noise in the megahertz frequency range emitted by collapsing bubbles close to the tip of the wire.

The 20-MHz probe is first driven in the pulse-echo mode and the ultrasonic wire is operated using a foot switch, the gain of the device being increased very gradually. Initially, no received signal at $20 \mathrm{MHz}$ can be detected. However, as the gain on the AUWS generator is increased, a small steady signal at the location of the $20-\mathrm{MHz}$ probe focus that is indicative of a bubble cloud forming at the tip of the wire can be detected. On increasing the gain on the wire generator further, the signal output at the probe increases in amplitude dramatically and becomes erratic in time. This response is indicative of violent collapse of bubbles, the sound resulting from which is being received by the probe at $20 \mathrm{MHz}$. Due to the continuous intense activity of bubble collapse at these gain settings of the generator, however, it is difficult to differentiate between the probe signal reflected from bubble clouds forming at the tip of the ball of the wire and the signal from violently collapsing bubbles. Therefore, the cavitation monitor is now switched to passive detection mode.

In passive mode, the probe transducer is not excited, functioning only as a narrow bandwidth receiver. The only

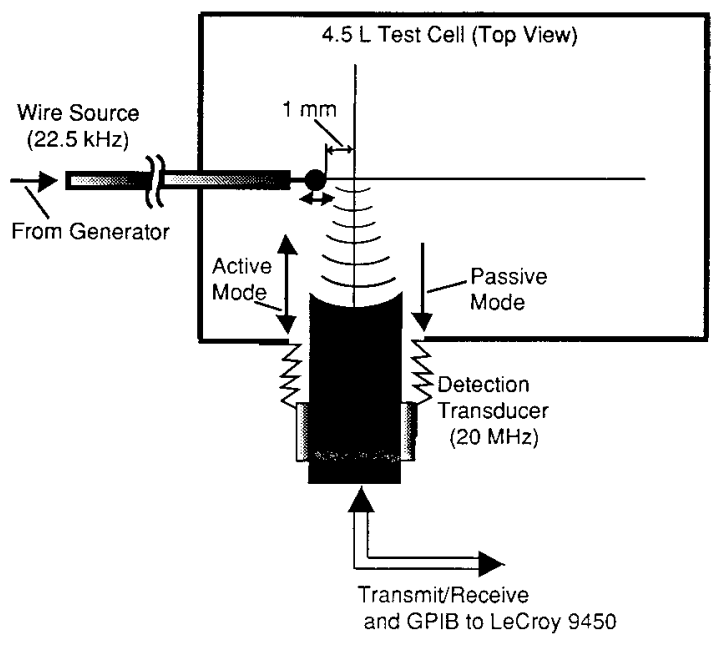

FIG. 6. Setup to measure cavitation during operation of the AUWS. 

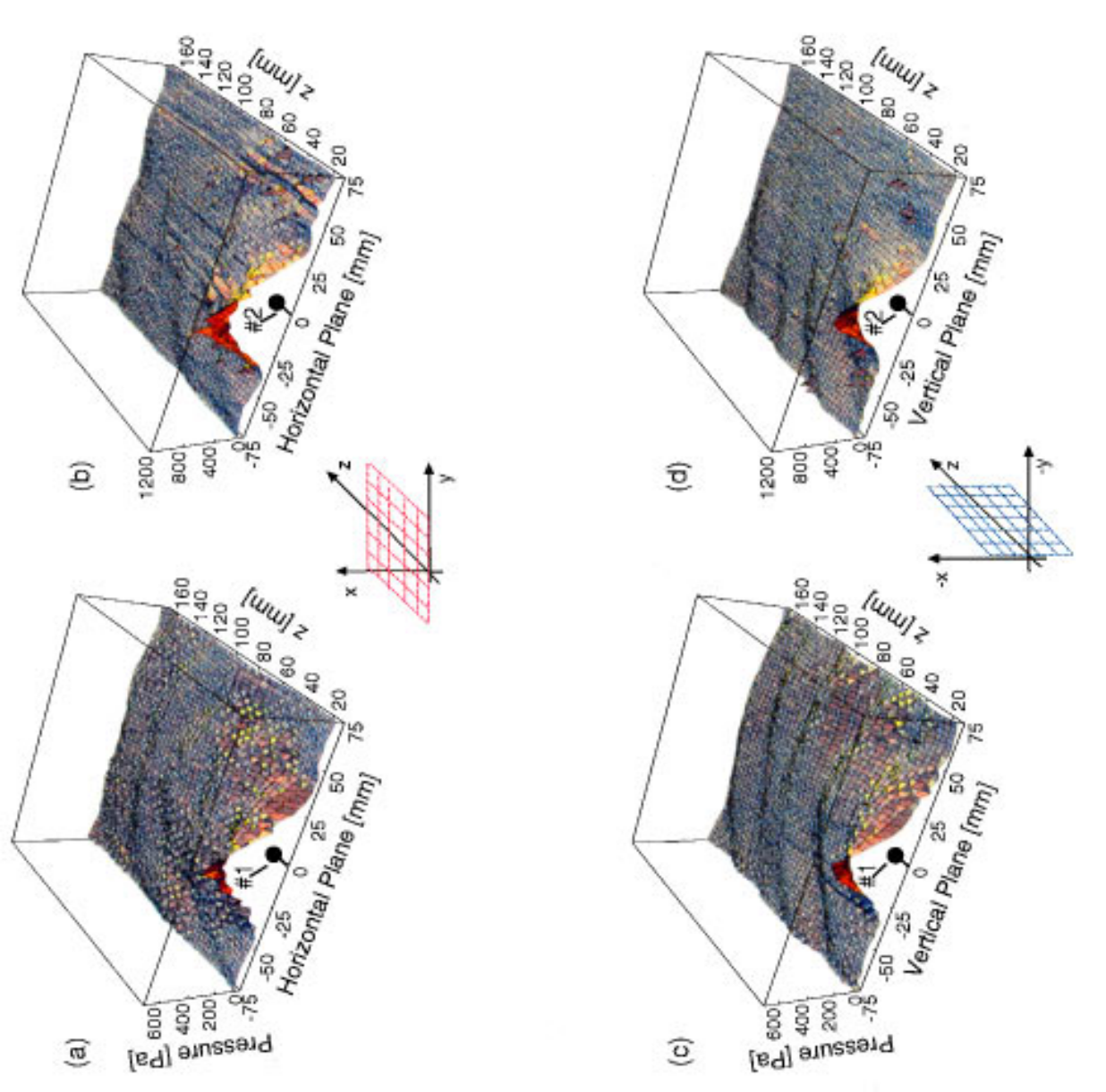

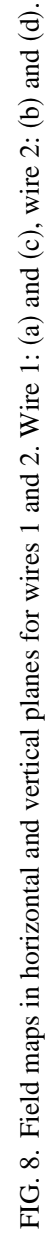
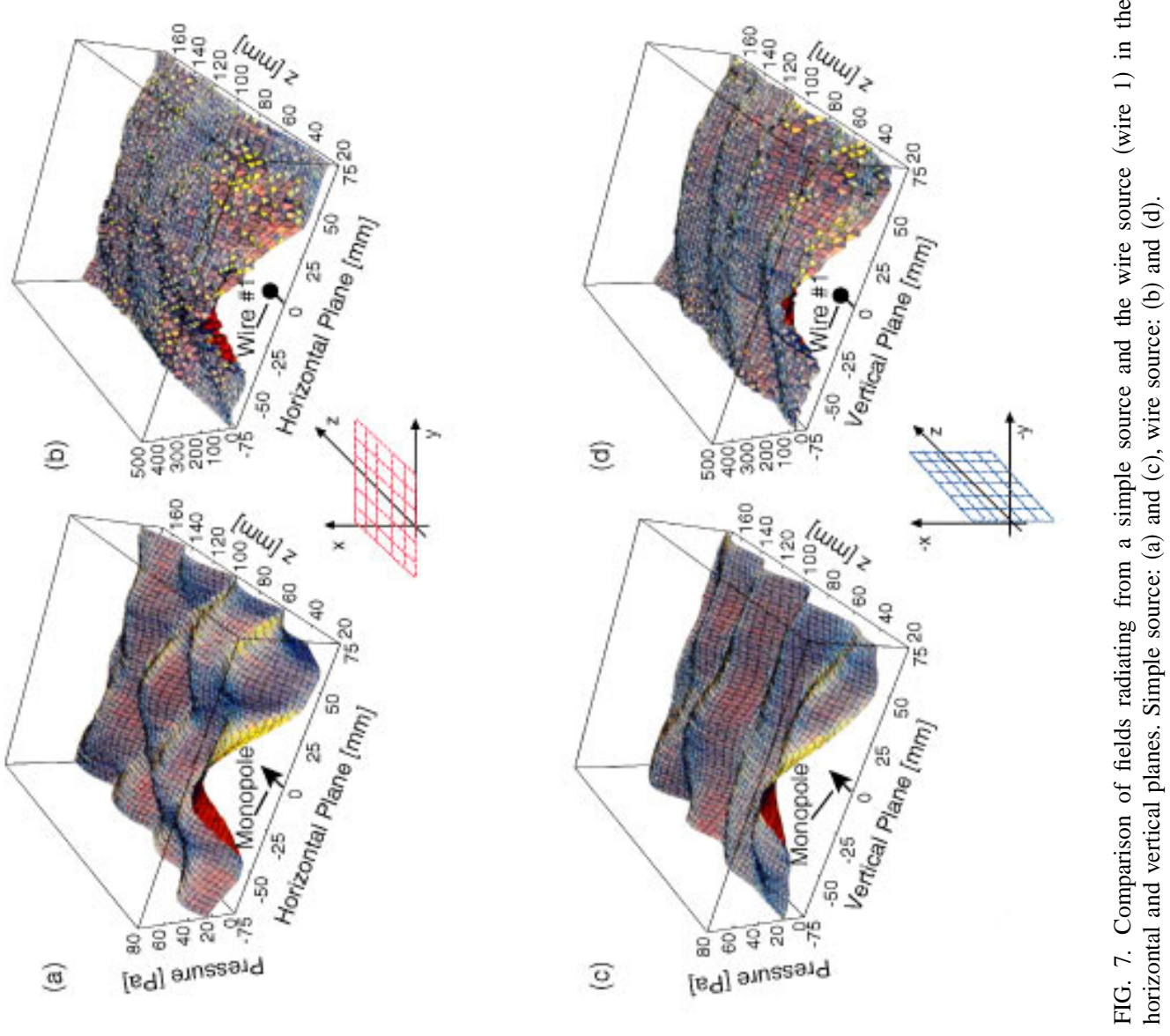

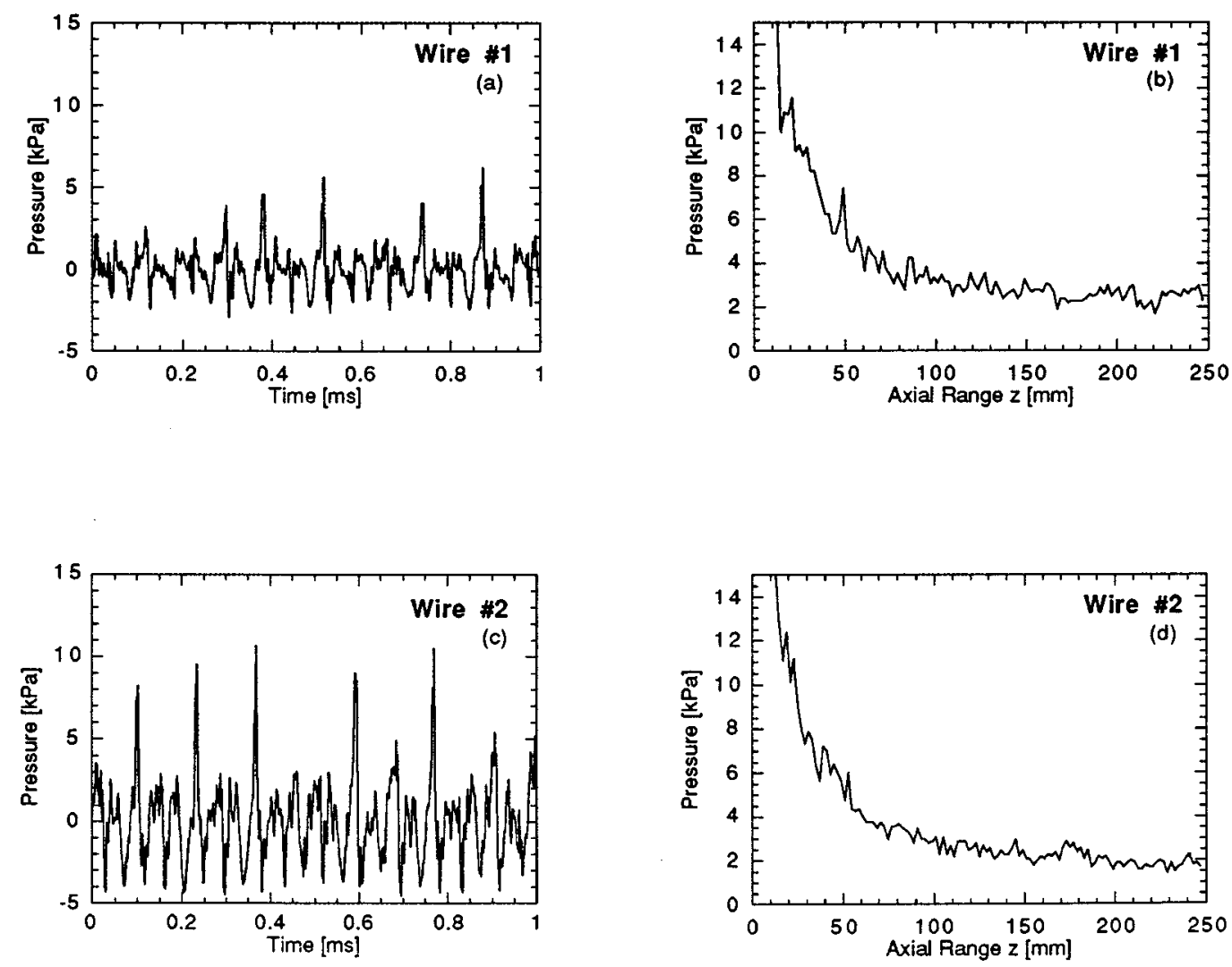

FIG. 9. Time waveforms [(a) and (c)], and axial field plots [(b) and (d)] for AUWS at high gain settings.

signal that can be observed at $20 \mathrm{MHz}$ in the current system is caused by the broadband signature of violent collapse of bubbles around the surface of the ball tip of the ultrasonic wire. The gain for the AUWS generator is maintained constant at a particular level. The device is then activated by the computer, and the acquisition of data from the $20 \mathrm{MHz}$ transducer is initiated after allowing the AUWS time (about $5 \mathrm{~s})$ to stabilize its output. The signal received as a result of cavitation at the ball tip is received by the $20-\mathrm{MHz}$ transducer, amplified by a broadband receiver amplifier (MATEC model 625), and displayed on a digital oscilloscope (LeCroy model 9450). A root-mean square (rms) value for each screen display is calculated by the oscilloscope. These rms values are downloaded to the computer 3 to 5 times per second and stored in a file. After a predetermined duration $(120 \mathrm{~s})$, the computer turns off the AUWS and simultaneously stops any further acquisition of data. Details of passive estimation of cavitation activity in an intense sound field with the help of a narrow bandwidth focused $20-\mathrm{MHz}$ probe is described by Everbach et al. ${ }^{14}$

\section{RESULTS}

\section{A. Acoustic field in a large volume}

As we described in Sec. I C, the field radiated by the AUWS is mapped in a relatively large tank, and is compared to the field from a simple source radiating in the $\mathrm{CW}$ mode in the same volume of fluid. Figure 7 shows a comparison of the fields in the horizontal and vertical planes, respectively, due to the two sources excited in the CW mode. The AUWS is operated at a low setting. We note a standing wave pattern in both cases due to reflections from boundaries of the tank. Important to note in these figures is the steeper decay of pressure with range in the case of AUWS source as compared to the simple source. The steep decay of pressure with range in the case of the AUWS source is indicative of its dipolelike radiation characteristic (note the pressure scales in Fig. 7). The pressure fields due to the wire source show a jaggedness as compared to the smooth simple source field. This jaggedness is caused by the transverse whipping of the wire even when it is operated at very low amplitude settings.

The field plots of the horizontal and vertical planes for wires 1 and 2 are shown in Fig. 8. Here the steep pressure decay close to the source becomes more apparent, since we now measure the field pressures starting closer to the source, i.e., from an axial range of $12 \mathrm{~mm}$. We observe higher pressures in Fig. 8(a) as compared to Fig. 8(b). The gain settings on the generator in both cases were kept approximately the same. Since the pressure radiated from a source is dependent on its size,,${ }^{10,11}$ the higher output from wire 2 as compared to 1 can be explained by its larger source dimensions (ball diameter of wire 2 is $2.46 \mathrm{~mm}$; wire 1 ball diameter is 1.98 $\mathrm{mm})$. Similar to the horizontal plane measurements, the pressure field plots for both wires in the vertical plane [Fig. 8(c) and (d)] initially decay rapidly, followed by a slower decay in pressure. Differences in the horizontal and vertical plane fields are because of variations in the tank boundary impedances.

At high gain settings of the device generator, as shown in Fig. 9(a) and (c), the time signature recorded at $13 \mathrm{~mm}$ 

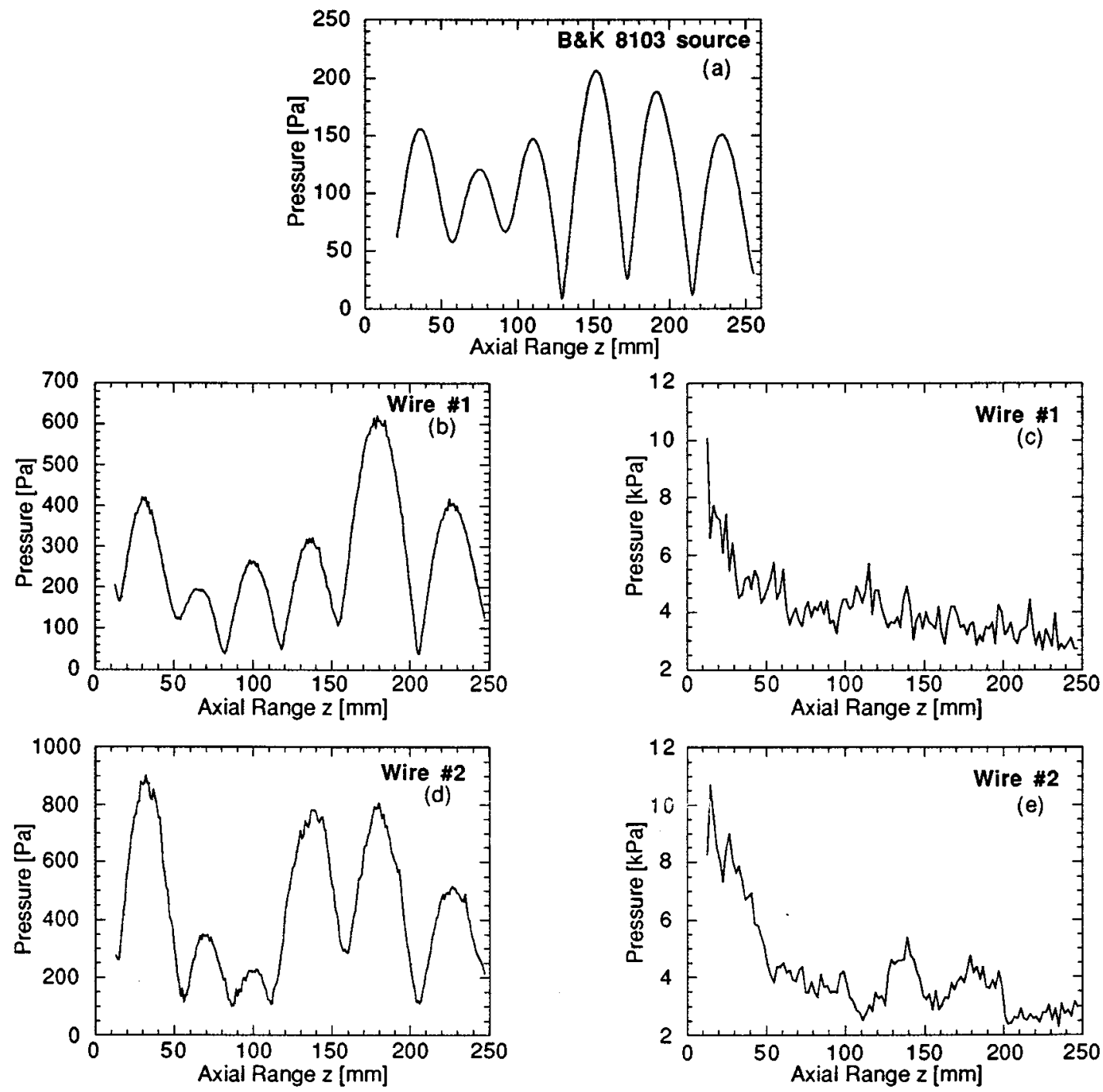

FIG. 10. Axial field plots with the source placed in a cylindrical tube.

from the wire tip is devoid of almost all periodicity and is quite complex. This complex signal is a result of the higher harmonics generated within the horn as well as the transverse whipping of the vibrating wire. In addition, significant jet formation is noted visually at the tip of the ball. The bubble activity further complicates the signal received at the hydrophone. Further, in Fig. 9(b) and (d) we note no standing wave pattern in the scans for peak pressures measured along the axis of the wire. On an average, the pressure is observed to decay monotonically with range. The acoustic pressures resulting at the surface of the ball tip extrapolated from the pressures measured on axis are at least a few atmospheres even with a conservative assumption of the pressure amplitudes varying inversely as range away from the wire source.

\section{B. Acoustic field in a cylindrical tube}

Measurement of the acoustic field radiated from the AUWS in a cylindrical space of about 11.5-liter capacity is a more appropriate approach in assessing the behavior of the device when it is operated within the human body. As de- scribed in Sec. I D, pressure measurements along the axis of the wire are made within a $12.5 \mathrm{~mm}$ polyurethane tube, inside which the ultrasonic wire is fixed.

Figure 10 shows the axial field plots for a water-filled cylinder with the central inner tube also filled with water. The source within the inner tube is a simple source vibrating at $22.5 \mathrm{kHz}$ in Fig. 10(a), the sources for plotting the axial fields in Fig. 10(b) and (d) are the ultrasonic wires 1 and 2 being operated at a low gain setting. We note a much more distinct standing wave pattern in this case as compared to the field plots mapped with the corresponding sources in a much larger volume as discussed in Sec. II A. In addition to the smaller volume of the cylinder compared to the large tank discussed earlier, the field here is further differentiable from that in Sec. II A due to the cylindrical shape of the enclosure.

Comparing the fields from a simple source in Fig. 10(a) to the field due to the dipole-like radiation of the ultrasonic wire in (b) and (d), we note some difference in the position of nulls between the two scans. The peak amplitudes are different for the two source types, however, but important to appreciate here is the limited axial range-dependent decay in 
the pressure amplitudes and a dominance of the standing wave pattern when a source is radiating at a frequency of $22.5 \mathrm{kHz}$ in water. On comparing Fig. 10(b) and (d), we note the output for the wire 2 to be higher than wire 1 for comparable gain settings of the generator. After removal of the central polyurethane tube and the top acrylic flange and repeating the scans described earlier in this section, no significant change in the standing wave pattern along the axis of the wire sources was observed (data not presented). Interfaces such as the 3.175-mm-thick acrylic walls of the cylinder are almost acoustically transparent to the $22.5-\mathrm{kHz}$ signal in water, and in fact the signal experiences an almost pressure release boundary at the interface between the air and the walls of the cylinder. This observation is of practical relevance to the use of the AUWS within the body.

The axial acoustic field plots obtained on operating the AUWS at high amplitude settings for the wires 1 and 2 are shown in Fig. 10(c) and (e). The time signature of the signal was complex as in the case of measurements in the large tank [Fig. 9(a) and (c)], and comprised of frequency components much higher than the $22.5-\mathrm{kHz}$ driving frequency. The peak pressure plots [Fig. 10(c) and (e)], show a much less significant standing wave pattern as compared to low gain settings of the device in (b) and (d). The less dominant standing wave character can be explained by the cancellation of the various modes, which are excited within the fluid volume when the source is radiating at multiple frequencies. The decay of pressure with range in this case, however, is not as marked as that observed in the corresponding measurements made in the large tank [Fig. 9(b) and (d)].

Finally, when the cylinder and the inside tube are filled with glycerin (results not presented here), we noted a marked decay of axial pressures with increasing range in addition to a standing wave pattern superimposed on the axial field. The normal modes which can be excited in the fluid filled cylinder now are different from those excited when it was filled with water, since the normal modes are a function of the sound speed of the medium. ${ }^{11,15}$ The decay of pressure in this case cannot be explained solely on the absorption of sound in glycerin. On pulsing the B\&K 8103 simple source at 22.5 $\mathrm{kHz}$ successively in water and the $66 \%$ glycerin fluid, no measurable difference between the fields in the two fluids could be measured within the sensitivity of the system. Further, the measured value for the absorption coefficient of $100 \%$ glycerin at $1 \mathrm{MHz}$ of $5.6 \mathrm{~Np} / \mathrm{m}$ by Averkiou, ${ }^{16}$ can be extrapolated to $22.5 \mathrm{kHz}$ by using an inverse quadratic frequency dependence and the value at $22.5 \mathrm{kHz}$ is 0.0028 $\mathrm{Np} / \mathrm{m}$. Since we are using $66 \%$ glycerin, the absorption for the fluid is expected to be even smaller, and hence negligible at this frequency.

\section{Measurement of cavitation}

Even at very low gain settings of the AUWS, microbubbles are observed to form at the tip of the vibrating ball. As described in Sec. I E, the bubble cloud is observed by scanning the region just beyond the ball tip with a 20$\mathrm{MHz}$ focused transducer and operated in the pulse-echo mode (data not shown).
For higher settings of the AUWS generator, when cavitational activity around the surface of the ball tip in the form of distinct bubble growth and collapse is established, the 20-MHz component of the sound due to collapse of bubbles is received by the detector transducer in the cell (described in Sec. IE and by Everbach et al.). ${ }^{14}$ Figure 11 shows the variation of the rms values of a $200-\mu$ s segment of the signal displayed on the oscilloscope. The rms data for each segment of the signal on the scope are downloaded to the computer 3 to 5 times per second for a total duration of $120 \mathrm{~s}$. The average activity of cavitation for each experimental run can be related to the mean of all downloaded rms values by the parameter $\overline{\mathrm{rms}}(\overline{\mathrm{rms}}=\langle\mathrm{rms}\rangle \times 1000)$. Comparing the plots for wire 1 in Fig. 11(a) and (c), we note a dependence of the cavitational activity at the ball tip on the settings of gain on the AUWS generator. Further, as can be seen by comparing the left and right-hand column plots in Fig. 11, the amount of cavitation occurring at the ball tip is dependent on the type of wire. Wire 1 is short, with a mushroom-shaped ball head, whereas wire 2 is longer with a larger, more spherical ball head. Cavitational activity is noted to be consistently higher at comparable gain settings for wire 1 as compared to the wire 2 . The effect of using different wire types at different generator settings is summarized in Fig. 11(e). This effect is in contrast to the measurement of pressures at low gain settings of the AUWS where the output from wire 1 was observed to be lower than from wire 2 .

\section{DISCUSSION AND SUMMARY}

The current study is motivated by the need to characterize a low-frequency intra-arterial angioplasty device currently under development for application in human patients. Since the device is driven acoustically, its effectiveness in practice could be enhanced by a better understanding of the manner in which the acoustic field generated by the device is distributed in a particular volume of fluid. Pressure measurements made with the wire device in a relatively large tank of water show a steeper rate of decay with range when compared with similar measurements made using a simple source, indicating a dipolelike radiation characteristic for AUWS. Further, as described in Sec. III B, both the wire sources as well as the simple source show a more marked standing wave pattern when operated in a small volume of fluid. The high standing wave ratio is a result of the long wavelength of the signal at $22.5 \mathrm{kHz}$ in tissuelike fluids compared to the dimensions of the excited fluid space, as well as the low absorption of sound at these frequencies. At such low driving frequencies, the inter organ interfaces having reasonably well matched impedances can be treated as acoustically transparent to the signal. The principal mechanism for standing waves in the body (i.e., if they do occur), is probably the acoustic field reflected from the human tissue-air interface. Acoustic pressure varies with range, the operating conditions of the AUWS such as the high gain settings at which the device is used in practice, as well as the $\rho c$ characteristics of the host medium.

Finally, based on calculations and measurements, the estimated pressures at the surface of the ball tip do result in 

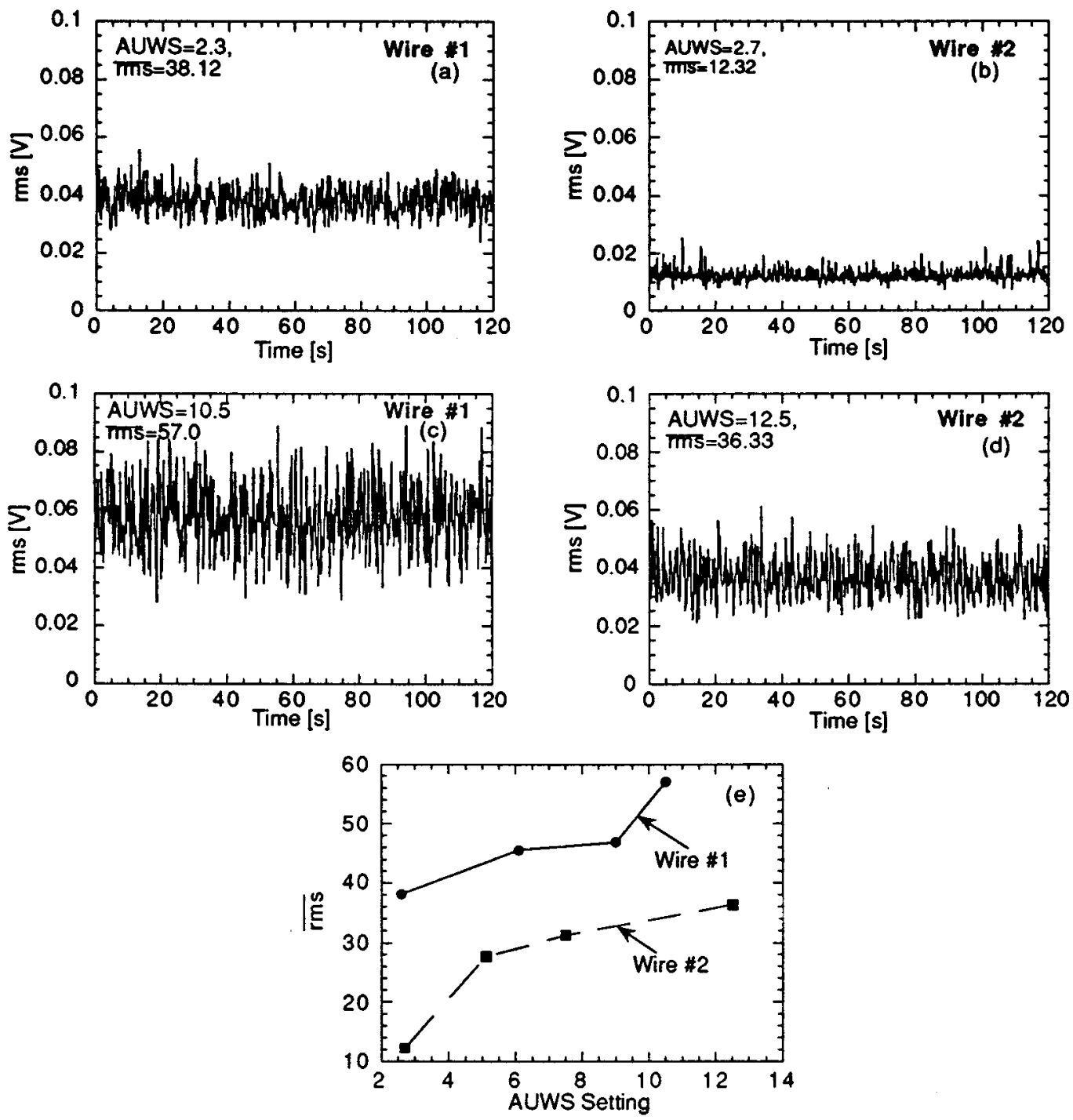

FIG. 11. Cavitational activity measured as the 20-MHz component of the sound due to bubble collapse, monitored over a $120-\mathrm{s}$ period from the two wire sources.

cavitation during operation of the device. In fact, cavitational activity has been hypothesized to be one of the principle mechanisms of action of such devices in causing disruption of atheromas. ${ }^{2,8}$ By operating the device at high gain settings, the occurrence of cavitation events based on the violent collapse of bubbles close to the surface of the ball tip was recorded. The amount of cavitation activity was measured for two representative wire tip configurations. The amount of cavitation due to the mushroom-shaped ball tip (1) was observed to be greater than the one having a more spherical shape (2), although the acoustic pressure amplitudes for the larger ball tip (2) were greater. The enhanced cavitation with the bluff mushroom-shaped wire tip as compared to the larger sized spherical wire is indicative of a hydrodynamic mode of occurrence of cavitation working in combination with cavitation due to the acoustic field. This issue has been briefly addressed in the Appendix. The occurrence of hydrodynamic cavitation probably becomes more significant at very high gain settings and for wire tip profiles with a sharper contour. Hence, if cavitation is indeed one of the principal mechanisms of action, then bluff shapes of ball tips could be expected to be more effective in a clinical setting than the smooth, spherical wire tips.

\section{ACKNOWLEDGMENTS}

This work was supported by an NSF Presidential Faculty Fellow award (Everbach), Number 92537777.

\section{APPENDIX: HYDRODYNAMIC CONSIDERATIONS}

In order to investigate the hydrodynamic phenomena, the ball tip is assumed to be spherical, the wire attachment at one end is ignored, and the movement of the spherical body is assumed to proceed in a non oscillatory manner, i.e., only along the $z$ axis (see Fig. 2 for coordinate system). Based on the maximum displacement of the ball tip $(\approx 100 \mu \mathrm{m})$, the maximum axial velocity is $v_{0}=14.14 \mathrm{~ms}$ at $f=22.5 \mathrm{kHz}$. The Reynolds number for the sphere can then be calculated as $^{17}$ 


$$
\operatorname{Re}=\frac{\rho v_{0} 2 a}{\mu}=4.13 \times 10^{4},
$$

where $a=1.3 \mathrm{~mm}$, and for water, density $\rho=1000 \mathrm{Kg} / \mathrm{m}^{3}$, and viscosity $\mu=0.89 \times 10^{-3} \mathrm{~N} \mathrm{~s} / \mathrm{m}^{2}$. For flow around a sphere, the Reynolds number calculated in Eq. (A1) is subcritical as compared to the high Reynolds number values which are associated with a sudden decrease in the drag coefficient. Therefore this flow is associated with a significantly high form drag coefficient. ${ }^{17,18}$ These drag forces result in a distribution of pressure magnitudes around the sphere much less than those predicted by theory for frictionless flow (about $1 \mathrm{~atm}$ ). ${ }^{17}$

Hydrodynamic cavitation occurs when the low fluid pressures are at or below the vapor pressure of the liquid. An estimate of the resistance of a flow system around a curved surface can be obtained by using the expression for the cavitation number $\sigma$ (also called Euler's number), which is defined as ${ }^{18-20}$

$$
\sigma=\frac{P_{0}-P_{v}}{\frac{1}{2} \rho v_{0}^{2}}=0.97,
$$

where $P_{0}=1 \mathrm{~atm}$ is the ambient static pressure, $P_{v}=0.03$ atm is the vapor pressure for water at $27{ }^{\circ} \mathrm{C} .{ }^{21}$ A relatively high value of $\sigma=0.97$ is indicative of inception of cavitation in the wake region of the sphere moving through water but not necessarily leading to violent cavitation ${ }^{19}$ as was observed physically with the device.

${ }^{1}$ B. F. Waller, “ 'Crackers, breakers, stretchers, drillers, scrapers, shavers, burners, welders, and melters'-The future treatment of atherosclerotic coronary artery disease? A clinical-morphological assessment,', J. Am. Coll. Cardiol. 13, 969-987 (1989).

${ }^{2}$ W. Steffen and R. J. Siegel, "Ultrasound Angioplasty-A review,' J. Interven. Cardiol. 6, 77-88 (1993).

${ }^{3}$ R. J. Siegel, D. C. Cumberland, and J. R. Crew, 'Ultrasound recanalization of diseased arteries,' Endovascular Surgery 72, 879-897 (1992).

${ }^{4}$ R. J. Siegel, P. Gaines, J. R. Crew, and D. C. Cumberland, “Clinical trial of percutaneous peripheral ultrasound angioplasty,' J. Am. Coll. Cardiol. 22, 480-4887 (1993).

${ }^{5}$ R. J. Siegel, J. Gunn, A. Khan, M. C. Fishbein, R. J. Bowes, D. Oakley, C. Wales, W. Steffen, S. Campbell, H. Nita, T. Mills, P. Silverton, R. K. Myler, and D. C. Cumberland, "Use of therapeutic ultrasound in percutaneous coronary angioplasty," Circulation 89, 1587-1592 (1993).

${ }^{6}$ U. Rosenschein, A. Frimerman, S. Laniado, and H. I. Miller, "Study of mechanism of ultrasound angioplasty from human thrombi and bovine aorta," Am. J. Cardiol. 22, 1263-1266 (1994).

${ }^{7}$ A. Ernst, E. A. Schenk, T. J. Woodlock, H. Alliger, S. Gottlieb, S. Z. Child, and R. S. Meltzer, "Feasibility of recanalization of human coronary arteries using high-intensity ultrasound," Am. J. Cardiol. 73, 126-132 (1994).

${ }^{8}$ W. L. Nyborg, "Basic physics of low-frequency therapeutic ultrasound," in Ultrasound Angioplasty, edited by R. Siegel (Kluwer Academic, Boston, 1996), pp. 1-23.

${ }^{9}$ I. R. S. Makin and E. C. Everbach, "Pressure and cavitation measurements of an intra-arterial angioplasty device," J. Acoust. Soc. Am. 97, 3326(A) (1995).

${ }^{10}$ D. T. Blackstock, "Class Notes" for a Course on Physical Acoustics (University of Texas at Austin, Austin, 1990) (unpublished).

${ }^{11}$ P. M. Morse, Vibration and Sound (American Institute of Physics, New York, 1986).

${ }^{12}$ R. A. Roy, S. I. Madanshetty, and R. E. Apfel, “An acoustic backscattering technique for the detection of transient cavitation produced by microsecond pulses of ultrasound," J. Acoust. Soc. Am. 87, 2451-2458 (1990).

${ }^{13}$ S. I. Madanshetty, R. A. Roy, and R. E. Apfel, “Acoustic microcavitation: its active and passive detection," J. Acoust. Soc. Am. 90, 1515-1526 (1991).

${ }^{14}$ E. C. Everbach, I. R. S. Makin, M. Azadniv, and R. S. Meltzer, "Correlation of ultrasound-induced hemolysis with cavitation detector output in vitro," Ultrasound Med. Biol. (in press).

${ }^{15}$ L. E. Kinsler, A. R. Frey, A. B. Coppens, and J. V. Sanders, Fundamentals of Acoustics (Wiley, New York, 1982).

${ }^{16} \mathrm{M}$. A. Averkiou, "Experimental investigation of propagation and reflection phenomena in finite amplitude beams," Ph.D. dissertation, The University of Texas at Austin, 1994.

${ }^{17}$ H. Schlichting, Boundary-Layer Theory (McGraw-Hill, New York, 1979).

${ }^{18}$ T. G. Leighton, The Acoustic Bubble (Academic, London, 1994).

${ }^{19}$ F. R. Young, Cavitation (McGraw-Hill, London, 1989).

${ }^{20}$ R. H. Sabersky and A. J. Acosta, Fluid Flow (Macmillan, New York, 1964).

${ }^{21}$ CRC Handbook of Tables for Applied Engineering Science, edited by R. E. Bolz and G. T. Tuve (CRC, Cleveland, 1973). 